\title{
Industrial Design and Simulation of a JIT Material Handling System
}

\author{
Chukwuedozie N. Ezema, Eric C. Okafor and Christiana C. Okezie
}

\begin{abstract}
A JIT Material Handling System attempts to manufacture products from start to finish by rearranging the factory floor layout away from batch production toward a product layout using flow lines. One of the most accepted JIT production methods used in this work is known as the Kanban system. Kanbans are used to operate a pull system of material control that links all supplying operations to a final assembly line. The ultimate goal of this system is the conversion of raw materials at the Juhel Oral Drug Process plant into finished products with lead time equal to processing time. The flows in the manufacturing model include: the flow of materials, the flow of information, and the flow of cost. These flows create the value streams. Components of the value stream can be valueadd or waste, depending on the operating conditions. The simulation model developed in this research work is based on the single-card pull system developed at the Drug Process Plant. This model consists of three blocks (workstations) where there is a buffer located between two workstations. In this model, the material moves according to the Kanban rule. If Kanbans arrive and the material is available, the workstation starts processing the material. This research demonstrates that the design of the JIT system can significantly affect key manufacturing performance measures. JIT implementation cannot provide significant benefits if setup time and variability remain high as well as if the company is not able to optimise the production facilities by product mix. Based on the result from this work, JIT is not based upon rigid concepts. Therefore, successful implementation depends largely on the company's ability to identify problems, creativity and efforts. The application of single-card pull system coupled with the use of the trigger point results in simpler mechanisms for operating the system.
\end{abstract}

Index Terms - Kanban system; System Flows;Pull Mechanisms; Trigger Point; Entity Flows

\section{INTRODUCTION}

According to [1], Just in Time (JIT) is a production system that emphasizes the application of JIT principles in the manufacture of products from raw materials to finished parts. JIT production is implemented by first reorganizing the factory floor layout from batch production to a product layout by means of flow lines. Such flow line could be Ushaped so as to allow workers help each other should there be any emergency, trouble or unpredicted occurrence or mishap in production line. It would also allow workers have easier access to more than one machine. The JIT system is time responsive and could shut down a whole production

Published on December 22, 2016

Chukwuedozie N. Ezema, Department of Electronic and Computer Engineering, Nnamdi Azikiwe University, Awka, Anambra State, Nigeria.

Eric C. Okafor, Department of Computer Engineering, Enugu State University of Science \& Technology, Enugu State, Nigeria.

Christiana C. Okezie, Department of Electronic and Computer Engineering, Nnamdi Azikiwe University, Awka, Anambra State, Nigeria. line in the emergence of a problem in production flow pending immediate fix of the problem. As obtainable in JIT, raw material are in constant flow since production is made based on demand. Under JIT environment, defects are nt tolerated. Minimum inventory levels are vigorously pursued as bottleneck in the production process may cause delays in product deliveries to customers. JIT production lay much emphasis on getting the job right the first time [2].

The Kanban system is one of the fundamental JIT concepts used in production. Kanban is a Japanese word for signal card and could be traced to the use of signal cards to manage a pull system of material control that connected all supplying operations to a final production assemblage line [3]. The utmost goal of the JIT system is to through the uses of Kanban match lead time with processing time as it converts raw materials into finished products. This is accomplished through inventory and lot size reduction, setup costs reduction, elimination of queues, WIP maintenance to eliminate defects, lead times reduction, effective collaboration with management and customers/vendors [4]. A cursory look at these factors in comparison with traditional manufacturing philosophy discloses why JIT implementation in Nigeria has been slow. Inventory management is vital in manufacturing plants, however traditional manufacturing plants strive to produce more ahead of demand $[2,5]$. Also, attempts are not usually made to reduce lots sizes, optimum lot sizes are determined using formulas based on the trade-off between inventories cost and setup cost. In traditional manufacturing plants, setup costs are not considered imperative because the firm's principal objective is to maximize output. Extra investments in queues become necessary and vendors are considered adversaries since competition between them is fostered. Scrap production and components defects are tolerated [1, 6]. Equipment repairs are done as necessary and it is not decisive because queues are available. Also, traditional manufacturing entertain longer lead time.

Furthermore, workers under traditional manufacturing environment make little or no input in the installation of new systems even though they are evaluated based on the new systems. The goals of a Kanban (JIT) system do not agree with the traditional manufacturing/management philosophies of conventional manufacturing companies. Kanban or JIT implementation involves considerable changes in the operating procedures/production processes of companies. According to [1], successful implementation of JIT requires the cooperation and commitment of both management and employees. JIT implementation in purchasing is dependent on the availability of raw materials from suppliers at the point they are needed by the company. Settings such as this require that a company must build up a 
very close relationship with its vendors in order for them to deliver quality raw materials on time.

\section{METHODOLOGY}

\section{A. Structure of the JIT Manufacturing System Model}

There are of three flows in the JIT manufacturing System model: materials flow, information flow, and cost flow. The value streams emanate from these flows. The value stream components can be value-add or waste, dependent on the operating environment. Excess material flows for instance become a stream of inventories, while excess information flows cause uncertainty in process execution. Proper management of the flows ensures effective control of the streams. To achieve lean production, an efficient control of these streams is essential.

As earlier stated, the flow is controlled by the interfaces. For instance a conveyor regulates materials flow and a visual control regulates the information flow between two stations. The interfaces arise from disconnected points in the system, as applicable to the physical distances between two machines, the control panels between a machine and an operator, the communication barriers between two people. It establishes a good location for cost transactions.

In the Just in Time Manufacturing System model, the parts and kanban represent the raw materials and the information mechanism respectively which are used to analyze the efficiency of these flows. Costs are applied to the operation of the devices that handles the parts or kanban. Therefore a buildup of parts and kanban lead to increase in cost.

Parts, kanban and cycles constitute the entities of the model. Parts produced in the production sub-model are consumed in the consumption sub-model and shipped from the production sub-model to the consumption sub-model. While on transit, they also travel through the supplier sub model and the plant sub model. The reordering of parts is controlled by Kanban. All kanban cards begin and terminate in the kanban sub-model whereas parts and kanban cards from the supplier sub-model are moved to the plant submodel. Cycle entities which exist only in the route submodel state the time to dispatch and signal the transport cycles.

\section{B. Material Handling System Flows}

Since, kanbans control the reordering of parts. The kanban cards flow pattern will be described below: A kanban card is dispatched in the kanban sub-model when inventory level hits a critical point. The card will be picked up at a specific time and moved to its designated supplier. The card is dropped off at the supplier. In order for the card to simulate the order-to-pickup lead time, it stays at supplier for a number of cycles. After that, another card is pulled out together alongside any available parts assigned to the card. The card is afterwards taken back to the plant and dropped off. The card is again dispatched to a HOLD block which accumulates all the extra cards.

The flow pattern of the parts is created at the supplier is as follows: Once a kanban signal is issued, a prototype part replicates another part. This occurs at the same instant the kanban card is issued for the kanban flow. The part is thereafter deferred in a process block to simulate production or dispensing. It then exits to a batch block and turn out to be part of a pallet. At a specific time, the pallet is picked up together with its kanban card and moved to the plant. At the plant the pallet is dropped off and taken to a holding block in the consumption sub-model.

\section{Purpose of the Evaluation of the New System}

The purpose of the evaluation of the JIT system using simulation is to determine factors contributing to improving performance of the new system. The design of the new JIT system was carried out in a more practical rather than theoretical manner. Therefore, some JIT characteristics such as the number of buffers, Kanban quantities and the number of Kanban at each block are determined using practical grounds. In this research work, four characteristics will be evaluated using simulation to achieve lower inventory and shorter flow time. These include:
a. The number of buffers
b. The location of buffers
c. Kanban quantities
d. The scheduling rule

\section{System Simulation}

The simulation model developed in this research work is based on the single-card pull system developed at the Drug Process Plant. This model consists of three blocks (workstations) where there is a buffer located between two workstations. In this model, the material moves according to the Kanban rule. Material processing is initiated at the workstation when Kanbans arrive and the material is on hand. Otherwise, if the material is not on hand, the Kanban waits until the buffer is refilled and the material is available. To run this model, in the start of the simulation, all buffers hold a particular number of materials as the initialization. Without this step, the simulation in no way takes place since the materials are not on hand.

In this research work, not all items produced by the Drug Process Plant will be simulated since there are around 97 periodical items of which the order quantities range from one sub-pallet to 700 sub-pallets. In the simulation model, several high-volume items are selected to represent the other Kanban items. The rest are represented by four hypothetical non-Kanban items that have total order volumes and total processing times the same as those represented. Three stages are required to model the JIT system at the Drug Process Plant:

a. Building the basic model and the animation.

b. Developing a more realistic model by extending the number of items represented and the parameters of the system.

c. Evaluating the actual system by increasing the number of buffers and Throughput.

\section{E. Building the Basic Model and Animation}

The objective of this stage is to develop the basic model and to verify the logic of the model. Item represented is only the trial item i.e. JBF 113104. The logic of this model can be established early due to its simplicity. The flow diagram describing this model is shown in Figure 1. 
As the program is simple and the number of entities existing in the system is small, the verification of the logic of the model, particularly the movement of the entities can be conducted easily using animation. In the animation, it is difficult to observe the movement of too many entities on the same screen.

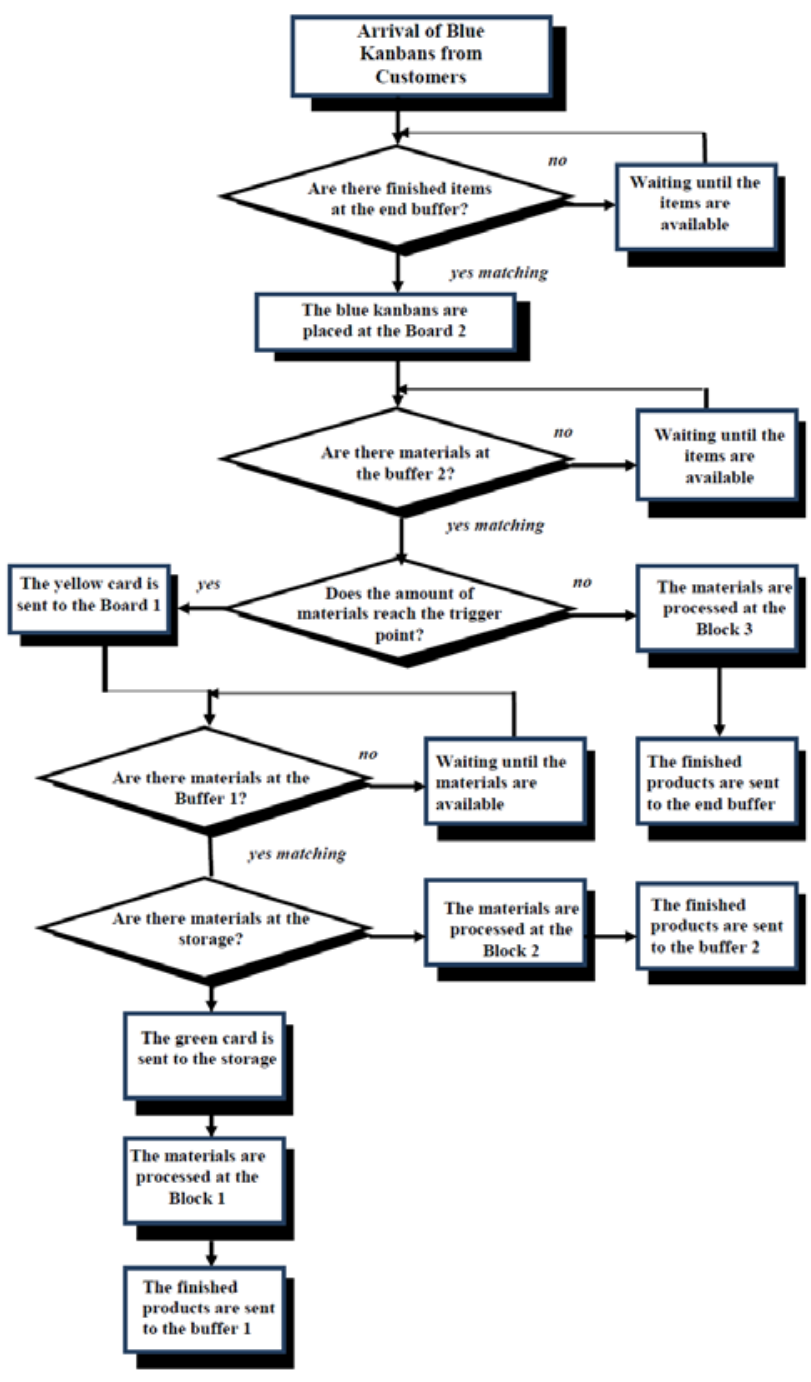

Fig. 1. The flow diagram for the model.

\section{Pull Mechanisms}

The pull mechanism is created by using MATCH, a SIMAN block, as suggested by [4]. Fundamentally, there are many ways of simulating the pull mechanism, however, MATCH has the advantage that this mechanism can be easily animated using SIMAN. Basically the purpose of MATCH is to synchronise two or more randomly arriving entities. In the model file, two randomly arriving entities that will be matched are Kanbans and materials. By using MATCH, the materials will be sent to the next process only if there is an entity represented as a Kanban staying in the other queue. As written in the model file, an example of this mechanism is as follows:

Board2 QUEUE, Board2Q:

DETACH;

Buff2 QUEUE, Buffer2Q:

DETACH;

MATCH, Buff2, Block3:

Board2;
Based on above model listing, materials at the queue buffer2Q will be sent to the Block 3 if there is a Kanban at the queue Board2Q. If a match does not occur either a Kanban or material stays in the queue pending when both of them are accessible.

\section{$\underline{\text { Trigger Point }}$}

A trigger point is also represented in this stage since block 3 and block 2 have different batch sizes. Based on this concept, buffer 2 will be refilled if the cumulative number of parts has reached the batch size of block 2 (360 units). To simulate the trigger point, an entity denoted by a green Kanban will pick up (match) the materials from buffer 2 . When the number of materials picked up reaches 60 units (represented by two entities) at the first trial, an entity represented by a yellow Kanban will be sent to block 2 to start producing 360 units of the new parts. If in the next trial, another entity denoted by a green Kanban arrives and the materials available at buffer 2 are more than 60 units, the yellow Kanban is not issued until the total requirement achieves 360 units or until the materials available are less than 60 units. This mechanism will ensure that there is a sufficient amount of materials consumed by block 3 and the pull system runs smoothly. In the model file, an example of this mechanism is shown as follows:

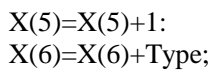

\section{BRANCH, 1:}

IF,(X(6).ge.2).and.(X(5).eq.1),label1:

IF,(X(6).ge.12).and.(X(5).eq.2),label1:

ELSE,label2; ! send a green Kanban to the Board 2 (normal) label1 ASSIGN X(6);

DUPLICATE: 1, Board1; !send a yellow Kanban to the preceding block or block 2

Based on the above model listing, a yellow Kanban will be sent to the workstation (Block) 2 denoted by the execution of label1, if the cumulative Kanban order (X(6)) achieves 60 units (or 2 entities, each entity representing 30 units) in the first trial $(X(5)=1)$, or if the $X(6)$ reaches 360 units (or 12 entities) in the next trial. Otherwise, if both conditions are not satisfied, label2 is being executed. That means green Kanbans move as usual to board 2.

\section{Determining the Arrivals of the Trial Item}

The arrival of the orders of JBF 113104 are daily, According to the order planning of the Plant. Therefore, if there are three shifts ( 24 hours), the arrival time is: $24 \times 60$ minutes $=1440$ minutes. If the difference of arrival time is around $10 \%$, this situation can be expressed in a statistical uniform distribution as UNIF(1440,1584).

The simulation model will be used to investigate the effect of the fluctuating orders. Although, the daily order in the trial period is constant, in the future it is likely to be unpredictable. The daily fluctuating order in the next six months can be illustrated as in the following Table 1 .

\begin{tabular}{|c|c|c|}
\hline TABLE I: PERCENTAGE OF ORDER QUANTITIES OF JBF 113104. \\
\hline ITEM & $\begin{array}{l}\text { ORDER } \\
\text { QUANTITY } \\
\text { (UNITS) }\end{array}$ & PERCENTAGE \\
\hline \multirow{3}{*}{ JBF 113104 (Trial) } & 30 & 10 \\
\cline { 2 - 3 } & 60 & 30 \\
\cline { 2 - 3 } & 90 & 50 \\
\cline { 2 - 3 } & 120 & 10 \\
\hline
\end{tabular}


Based on the Table 1, the arrival and the amount of the order quantities can be written in the model file as follows:

CREATE : UNIF(1440,1584,2):

MARK(Arrtime1);

ASSIGN : Type=DISC(0.1,1,0.4,2,0.9,3,1.0,4); ! Arrivals of Green Kanbans

\section{Entity Flows}

SIMAN is designed for the conventional push system, therefore, to simulate a pull system, statement DUPLICATE will be utilized to send entities to the opposite direction in the push system. In each block, entities (materials) wait at each station according to the processing time. An entity must move to the buffer located at the subsequent block, after being processed at the station. Hence, a DUPLICATE should be used to move the entity in the reverse direction. In the same way, this approach is used to fire a signal or a Kanban to the preceding workstation. The original entities remain in the workstation for counting and after that they are disposed off.

\section{Animation}

The animatronics developed in this stage presents displays about the model such as the movement of entities, the amount of the buffers as well as the level of the orders queue. The movement of entities such as Kanbans and materials are animated. This display therefore offers a useful means to verify the logic of the model. To get insight into the performance of the system, the status of the parameters such as the amount of each buffer as well as the number of items produced at each block can also be observed. In addition, the animation also shows the histogram of the queuing orders so the level of unsatisfied orders can also be observed. The animation screen can be seen in Figure 2 .

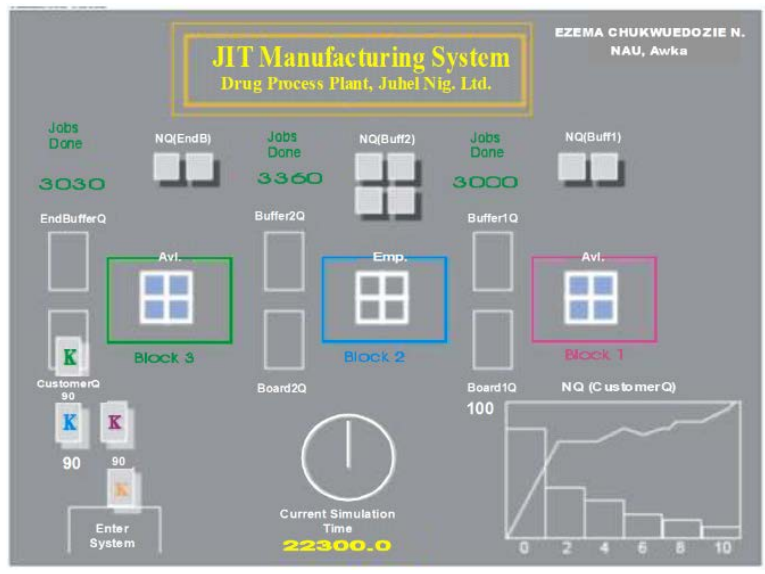

Fig. 2. Animation Screen.

\section{RESULTS}

\section{A. Behavior Analysis of the Production Control}

A plot of the production rate and total inventory level on the 'I1 - I2' plane are shown in Figure 3 and Figure 4 respectively where I1 and I2 are the loop invariants.

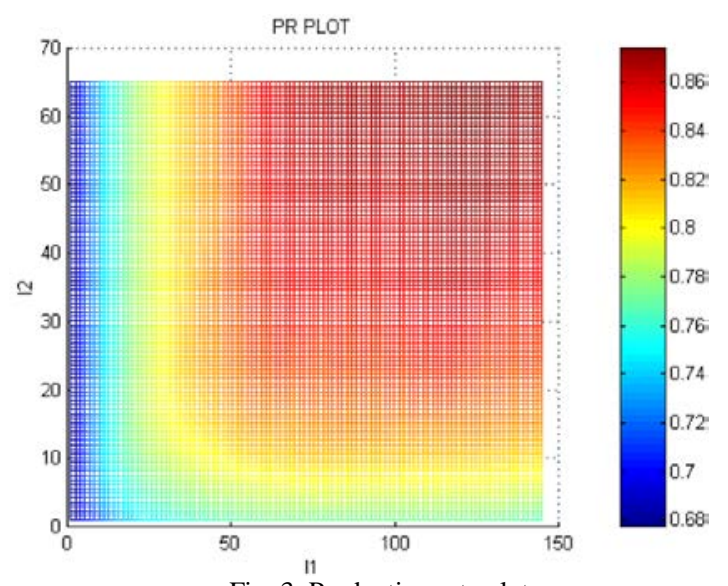

Fig. 3. Production rate plot.

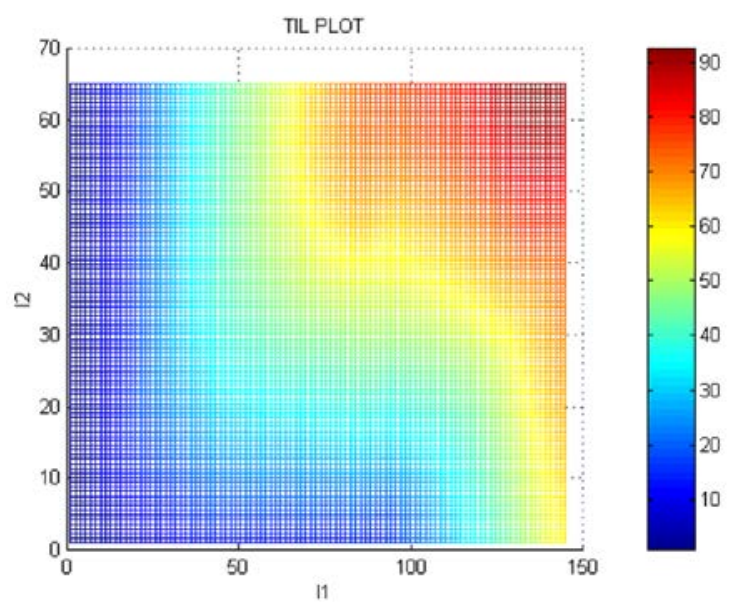

Fig. 4. Total inventory level plot.

Production rate plots provide a platform in which isocurves can be drawn. Points on the production rate iso-curve match with a combination of $\left(I_{1}, I_{2}\right)$ that corresponds to a specific production rate. It is important to state that the production rate plot is identical to the production plot for the buffer space allocation.

These arrangements have different values in the total inventory level plot. As a rule, the solution of optimizing total inventory level is the one that has the least value [7]. In Figure 5 and Figure 6, this paper illustrates an iso-curve mapping technique to establish the loop invariants which optimize total inventory level.

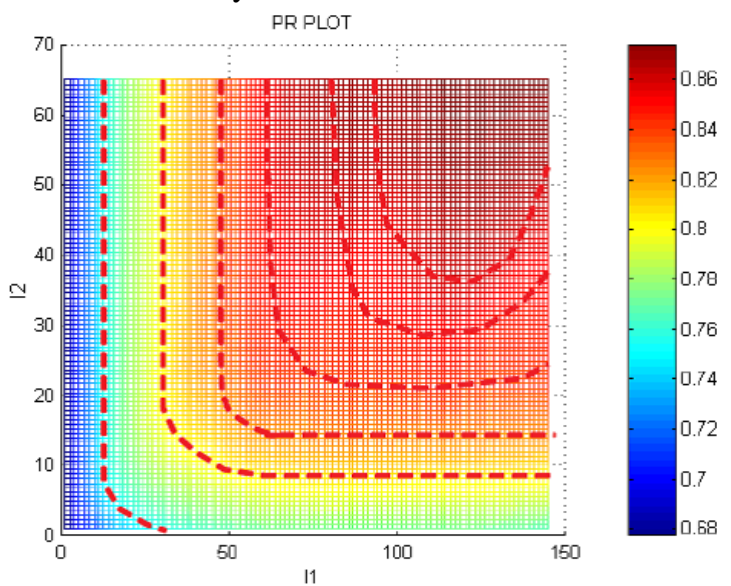

Fig. 5. Optimal loop invariants of the loop control. 


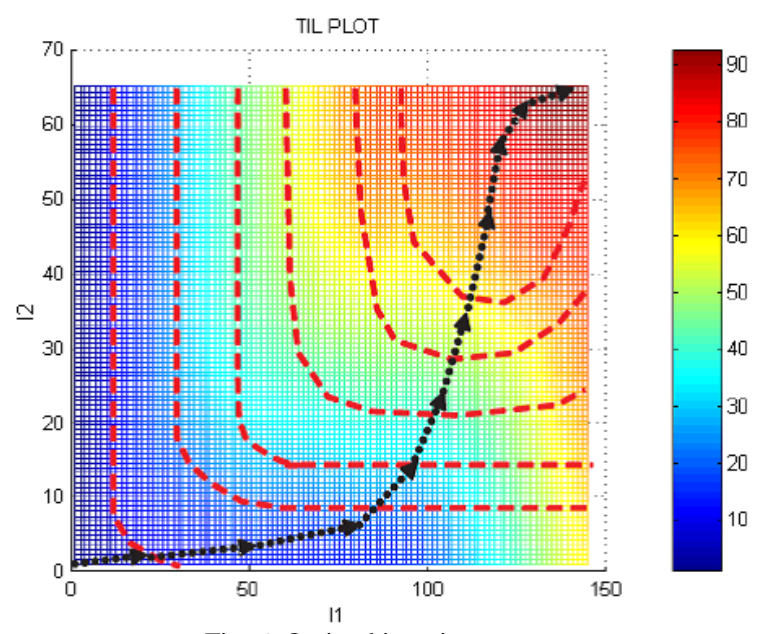

Fig. 6. Optimal invariant curve.

\section{B. Sensitivity Analysis}

The total inventory cost function is a picture of the real solutions in which the model parameters (total demand of finished product, finished product demand changing rate, ordering cost, holding cost, etc) are understood to be static values. It is logical to study the sensitivity, i.e. the effects of making changes in the model parameters over a given optimum solution. Numerical sensitivity of the system parameters and input variables are evaluated in this section. The analysis shows the wide-ranging behavior of the system and illustrates the characteristics of the parameters through the nature of the curvature. The result gives the sensitivity of the model parameters on the total inventory cost and demonstrates the critical point for the cost minimization.

\section{Effect of Finished Product Demand on the Inventory Cost at Different Raw Material Orders}

In a JIT-Kanban based production system, the finished product demand $\left(D_{F}\right)$ is a vital factor. Finished product demand establish the on-hand inventory, especially when finished product demand shifts significantly influence the overall inventory cost. Therefore, it is crucial to perform a sensitivity analysis based on the variation of finished product demand. Keeping the other parameters of the total inventory cost function unchanged, the effect of $D_{F}$ over the total inventory cost is shown in Figure 7 . It is noted that when the demand of finished product increase, the total inventory cost also increase in a linear fashion, and the optimal raw material orders increases to some extent.

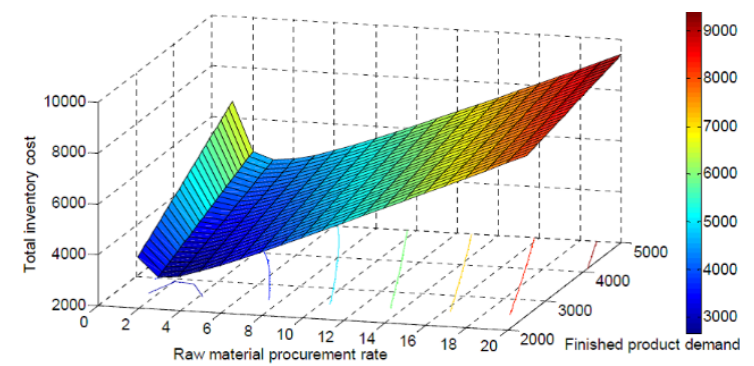

Fig. 7. Effect of finished product demand on total inventory cost and raw material procurement rate.

\section{Effect of finished product demand rate on the total}

inventory cost at different raw material orders

Figure 8 shows the finished product demand changing rate and raw material procurement rate VS the total inventory cost by applying the parametric values and varying the raw material procurement rate from 1 to 20 and finished product demand changing rate from 4 to 20. It is observed that when the demand changing rate of finished product rises, the total inventory cost is decreased inversely but the optimal raw material orders rises.

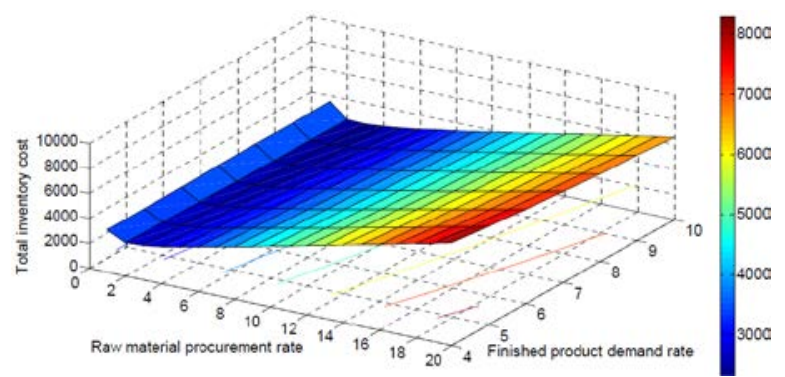

Fig. 8. Effect of finished product demand rate on both total inventory cos and raw material procurement rate.

\section{E. Effect of JIT system on Key Manufacturing Performance Measures}

Findings from the plots reveal that JIT system design can extensively influence key manufacturing, market, operational, and financial performance measures like demand fulfillment rate (DFR), cycle-time (CT), and net operating income (NOI). This confirms the findings of $[8,9$, $10]$.

\section{CONCLUSION}

The Just-In-Time (JIT) system implemented at the Drug Process Plant provides many benefits, particularly those associated with inventory reduction at the end buffer, shorter customer lead time, better visual control and better worker motivation. However, for the reason that this implementation was only a pilot project and it was applied to only one item, problems arose in the implementation that may not completely reflect the real problems encountered in the implementation for multiple items. Some issues such as setup time reduction, process variability reduction and product mix that are essential for successful JIT implementation did not become crucial issues in this research. JIT implementation cannot offer significant profit if setup time and variability remain high as well as if the company is not able to optimise the production facilities by product mix. However, in the future, those issues should be taken into account if the Drug Process Plant wants to expand the system for use with other items.

Based on experience from this research work, JIT is not based upon rigid concepts. Therefore, successful implementation depends largely on the company's ability to spot problems, their creativity and efforts. The single-card pull system applied in this project coupled with the use of the trigger point results in simpler mechanisms for operating the system. This modification means less containers or boxes are required and there is easier understanding for the operators. Basically, each company can develop their own pull system. However, the most important thing is how making the system work and meeting the requirements. 


\section{REFERENCES}

[1] Keng, H.C., 2004. Optimization and Simulation of Just-in-Time Supply Pickup and Delivery Systems. University of Kentucky, Lexington.

[2] Neumann, B. R., Jaouen, P. R., 2016. Kanban, Zips and Cost Accounting: A Case Study. Journal of Accountancy. 35, 132-141.

[3] Papadopoulus, H. T., Heavey, C., Browne, J., 2013. Queueing Theory in Manufacturing Systems Analysis and Design.Chapman and Hall. 33, 345-375

[4] Pegden, C., Denis, R.E., Randall, P., 2011. Introduction to Simulation Using SIMAN.McGraw-Hill Book Co. Second Ed. 12, 9-25.

[5] Robinson, M.A., Timmerman, J.E., 2014. How vendor analysis supports JIT. Management Accounting. 5, 20-24.

[6] Hassan, Y., Belal B., Ed Hsu C., 2007. A review of the adoption of JIT method and its effect on efficiency. PAMIJ. 12, 2

[7] Zhenyu, Z., 2006. Analysis and Design of Manufacturing Systems with Multiple-Loop Structures. Massachusetts Institute of Technology, Cambridge

[8] Robert, H., 2007. The Impact of Time Based Accounting on Manufacturing Performance. University of Toledo, Ohio.

[9] Svensson, S., 2001. Just-in-Time: the reincarnation of past theory and practice. Management Decision. 39, 866-879.

[10]Doll, W. J., Vondermbse, M. A., 2011. The evolution of manufacturing systems: towards the post-industrial enterprise. OMEGA: International Journal of Management Science. 19, 401-411. 